\title{
TÓPICA Y DIALÉCTICA EN LA LÓGICA MARXIANA DE LA INVESTIGACIÓN
}

1. Fue Engels el primero que, aún antes de Marx mismo, planteó la cuestión del método de Marx y de su relación con el de Hegel. En la reseña a la Critica de la economia política aparecida en el periódico Das Volk, editado por Marx en Londres, en agosto de 1859, luego de un largo análisis de la dialéctica hegeliana, enuncia por primera vez lo que luego, repetido por Marx en el prefacio a la segunda edición del Capital (1873), se convertirá en el lugar común del método marxista. La cita de Engels dice asf:

...el desarrollo de su pensamiento (de Hegel) corria siempre paralelamente al desarrollo de la historia universal, y el último debfa ser en sentido propio sólo la prueba del primero. Si bien de este modo la relación correcta estaba invertida y puesta de cabeza, aún asi se introdujo por todas partes en la filosofía... Marx fue y es el único que podía tomarse el trabajo de extraer el núcleo de la lógica hegeliana, núcleo que comprende los verdaderos descubrimientos de Hegel en este ámbito, y de reponer al método dialéctico, desnudado de sus envolturas idealistas, en la forma simple en la que este método se convierte en la única forma correcta del desarrollo del pensamiento. La elaboración del método sobre la que se apoya la Critica de la economia politica de Marx, constituye para nosotros un resultado apenas inferior en significación a la concepción materialista fundamental. ${ }^{1}$

Señalemos dos particularidades de esta exaltación del método: (a) escrita en una época aún próxima a la Ideología alemana (1846), época en la que él contribuía aún de modo activo y creador a la superación positiva del hegelianismo en la concepción de la historia, Engels se limita a plantear la cuestión del método en el ámbito específico de la lógica y de la historia, esto es, en el plano estricto de lo que luego quedará acuñado (y tergiversado) bajo el rótulo de "materialismo histórico". Lejos está aún de sus especulaciones cientúfico-naturalistas posteriores, que tanta confusión introdujeron. (b) La cuestión del método se restringe, según Engels, a la conexión entre los hechos históricos y su evolución, por una parte, y las categorias conceptuales que aprehenden a ambos, por otra. Trasladado esto al plano especifico de la materia tratada, la economfa, el método en cuestión es el que Marx emplea para la exposición concatenada de los distintos fenómenos económicos: mercancía, valor, valor de cambio, dinero, etc. ${ }^{2}$

Demos un salto en la historia de esta progresiva apoteosis del método y

1 MEW 13, pp. 473-474.

2 Cf. op. cit., pp. 475-477. 
analicemos una nota redactada por Lenin en el año 1915 y recogida luego por primera vez en la edición de sus Obras Completas. La nota, que entretanto se hizo famosa, dice así:

Si Marx no nos dejó una "Lógica", dejó en cambio la lógica del Capital... En el Capital, Marx aplicó a una sola ciencia la lógica, la dialéctica y la teoría del conocimiento del materialismo... que tomó todo lo que habia de valioso en Hegel y lo desarrolló. ${ }^{3}$

El núcleo de la afirmación de Lenin es el mismo que encontramos en la reseña de Engels, sólo que el punto de vista ha variado un poco, y sin duda para peor. En lugar de la fluida y problemática relación entre lógica e historia, encontramos aquí la aplicación de un sistema cerrado, fijo y abstracto de lógica, dialéctica y teoría del conocimiento a un pequeño ámbito especial, la teoría económica, del universo de cosas posibles al que virtualmente podría extenderse. No es de extrañar, por lo tanto, que el pensamiento soviético diera fácilmente este paso. Así Rosental, partiendo de la afirmación leninista, afirma que, luego de haber elaborado el método, Marx "necesitaba 'solamente' aplicarlo a la economía politica". El Capital, agrega más adelante, "cuyo objeto inmediato de investigación no es la filosofía... expone sin embargo una 'ciencia de la lógica' en el verdadero sentido de la palabra"; "...El Capital (en efecto) corporiza de modo evidente la indisoluble unión de dialéctica y explicación materialista del mundo. Marx pudo justamente por ello crear y fundamentar el método dialéctico desde un punto de vista verdaderamente científico, porque vio la conexión orgánica de este método con el materialismo filosófico". ${ }^{4}$ La extensión del método como sistema al universo de objetos a través de la introducción subrepticia del "materialismo filosofico" encuentra aquí su culminación. El método, como Cronos, ha tragado a su hijo, El Capital, para perpetuarse él mismo en su infinita inmovilidad.

2. A estas afirmaciones metodológicas de Engels y sus epígonos vamos a oponer un conjunto de observaciones provenientes de la densa correspondencia que se cruzaba entre Marx, en Londres, y Engels en Manchester por la época en que el primero daba la última mano a la Critica de la economia politica, antes de enviarla al editor F. Duncker de Berlín. La primera data de enero de 1858 y dice así:

En el método de la elaboración me ha prestado un gran servicio el hecho de que, por mero accidente - Freiligrath encontró algunos tomos de Hegel, que originalmente pertenecieron a Bakunin, y me los envio como presente- haya hojeado nuevamente la "Lógica" de Hegel. Si alguna vez tengo nuevamente tiempo para tales trabajos, tendría gran placer en hacer accesible al entendimiento del hombre común en dos o

3 V. I. Lenin, Cuadernos Filosóficos, Ob. com. t. 38 (Buenos Aires: Cartago, 1960), p. 311.

4 M. M. Rosental, Die dialektische Methode der politischen ökonomie von Karl Marx, Berlin: Dietz, 1969, pp. 10-14. 
tres folios lo racional en el método que Hegel ha descubierto pero mistificado al mismo tiempo. 5

Se imponen de inmediato dos observaciones: (a) la elaboración de que habla Marx debe referirse a la redacción final de su trabajo, esto es, a la disposición definitiva de un material científico ya previamente acumulado e investigado. Este material, el cuerpo mismo de la investigación con sus hipótesis y su recolección de datos, no pudo haber esperado el "mere accident" de haber recibido como regalo la Lógica de Hegel para ver la luz. Estaba por tanto ya realizado - constituye, por lo demás, el enorme volumen de 1000 páginas editado en el año 1939 como los Grundrisse-, cuando Marx lo sometió a una última elaboración a fin de darlo a publicidad. Es en ese momento, en el momento de fijar el esquema general de la obra, esquema que resume su concepción de la totalidad del sistema económico del capitalismo, en el que la relectura de la Lógica de Hegel, con su rigurosa concatenación de conceptos, contribuye decisivamente a que el esquema quede formado como una totalidad dialéctica. Poseemos al menos tres redacciones de ese esquema, dos muy breves en los Grundrisse ${ }^{6}$ y una bastante más extensa en una carta a Engels del año 1858. ${ }^{7}$ Las tres, sobre todo la última, confirman la diferencia entre investigación y exposición que se desprende de esta carta. (b) Para Marx el campo propio de la Lógica y el de la economía política estaban claramente deslindados. De ninguna manera pretende haber expuesto en sus escritos económicos el núcleo racional de la lógica hegeliana, dado que esta tarea correspondía a un tipo totalmente distinto de actividad, que él llevó a cabo en su juventud y que con gusto volvería a hacer: la reflexión filosófica. La cuestión de la relación entre la dialéctica hegeliana y la marxiana queda aún abierta.

Sobre esta relación arroja luz otra carta de Marx, posterior en quince dias a la que acabamos de citar, en la que éste comenta a Engels con frases lapidarias una nota de la obra de F. Lassalle, Herakleitos der Dunkel. Lassalle se refiere al tragmento 90 (Diels-Kranz) de Heráclito ". . como el oro se transforma en todas las cosas y todas las cosas en oro".

La cita de Marx es la siguiente:

El (Lasalle) utiliza esta sorprendente opinión para darnos en una larga nota una muestra de sus descubrimientos en la ciencia de la economía política. Cada palabra un error, pero expuesto con asombrosa pretensión. De esta única nota veo que el fulano tiene intenciones de exponer la economía política hegelianamente en su segunda gran obra. Para su perjuicio aprenderá que es una cosa totalmente distinta llevar una ciencia por medio de la critica al punto a partir del cual se la puede exponer dialécticamente, o (=que) aplicar un sistema de lógica, abstracto y ya hecho, a los balbuceos precisamente de un sistema como ése. ${ }^{8}$

5 Marx a Engels, 16.1.1858, MEW 29, p. 260.

6 Moskau 1939-41; rep. fotom. Frankfurt-Wien, s.f., pp. 28-29 y 175.

7 Carta a Engels, 2.4.1858, MEW 29, pp. 311 ss.

8 Marx a Engels, 1.2.1858, $M E W$ 29, p. 275. 
Con respecto a la misma nota escribe un año después:

...él debe... advertir, ... que él (Lasalle) es un burro, que pretende, con un par de frases abstractas como 'unidad abstracta' y semejantes, juzgar sobre cosas empiricas, que se deben estudiar, y con creces, para poder hablar sobre ellas. ${ }^{8}$

De ambas citas surge con claridad: (a) Marx condena como mera improvisación sin seriedad cientffica alguna la aplicación justamente de un "sistema de lógica, abstracto y ya hecho" a cosas empíricas, como las de la economía política, que exigen un largo proceso de investigación para poder dominarlas. (b) Este largo proceso de investigación y de apropiación del material emplrico es lo que Marx distingue como la tarea crítica en la elaboración de una ciencia, en lo que consiste la verdadera tarea de investigación, el verdadero método investigativo. (c) Sólo cuando se ha llegado al punto de dominar crfticamente el conjunto de datos e hipótesis propias y ajenas, puede exponerse la ciencia dialécticamente, esto es, siguiendo categorfas generales y desarrollando los conceptos.

Lo que Marx distingue aquí son dos procedimientos denominados por él con dos términos también diferentes: mientras que reserva la denominación de "dialéctica" al sistema general de conceptos concatenados contenido en la Lógica de Hegel, señala como el verdadero método cientffico a la "crítica", es decir, al método elaborado por los jóvenes hegelianos, en especial por L. Feuerbach. El espíritu de este método era el de volver contra la misma filosofía especulativa el arma de la dialéctica, no simplemente para refutarla, sino para extraer de ella la verdad oculta. ${ }^{10}$ Para ello Feuerbach se remonta al examen de la misma predicación: en el juicio teológico, en efecto, se ha invertido el orden, esto es, se ha supuesto una esencia divina como sujeto al cual se le atribuyen los predicados propiamente humanos. Invirtiendo el orden del juicio, poniendo como sujeto los predicados, obtenemos la verdadera for$\mathrm{ma}$, la forma antropológica de la religión. ${ }^{11} \mathrm{Al}$ poner bajo examen el juicio de esencia, esto es, aquel que encierra la definición de un sujeto, Feuerbach se remonta de hecho a una forma de la dialéctica vigente aún en la tradición alemana en la época de Kant, que en última instancia proviene del mismo Aristóteles. Se trata de la dialéctica entendida por oposición a la analítica o silogística como la parte de la lógica que trata tanto de los razonamientos hipotéticos como de la refutación y, en última instancia, del establecimiento de los primeros principios, de los cuales habrán de derivarse las primeras premisas o axiomas de la ciencia. Éste era propiamente el tema de los Tópicos de Aristóteles, cuya vigencia se habła mantenido en Europa continental hasta la difusión del ramismo. ${ }^{12}$ Retomada luego por los aristotélicos alemanes, la

9 Marx a Engels, 25.2.1859, MEW 29, p. 404.

10 L. Feuerbach, Das Wesen des Christentums, Stuttgart: Reclam, 1969, pp. 5-12.

11 Op. cit., prólogo a la $2^{2}$ edición, pp. 23 ss.

12 Para la definición de dialéctica en Aristóteles: Top, I 2, 191 a 134 ss.; P. Wilpert, "Aristoteles und die Dialektik", Kant-Studien 48 (1956/57) pp. 247-257; para la dialéctica en la época moderna antes de Kant, G. Tonelli, "Der historische Ursprung der kantischen 
distinción entre analítica y dialéctica será reconocida hasta la época del mismo Kant. ${ }^{13}$ Desalojada, empero, de la filosofía teórica por el pensamiento axiomático-deductivo inspirado en las matemáticas desde el cartesianismo en adelante, la tópica como método crítico de razonamiento y prueba se mantuvo, aunque jaqueada por el nuevo ideal de ciencia, allí donde se conservó la tradición de la antigua filosofía práctica. ${ }^{14}$ Es típico de la enorme complejidad del pensamiento hegeliano el hecho de que, por una parte, Hegel se asocie a la antigua tópica contra el método dogmático deductivo, inspirado en la matemática, de la metafísica racionalista, y por otro, conciba a la dialéctica como una unidad de la multiplicidad, acercándose con ello, real o aparentemente, al intento de la metafísica racional por él impugnada, de concebir la totalidad del mundo en un sistema de categorias. ${ }^{15}$

Con ello aparece con mayor claridad la significación que tiene en el joven Marx el desarrollo de la critica tras las huellas de Feuerbach, en especial de la critica a.Hegel. En la práctica equivalía a oponer en el interior de la dialéctica hegeliana el aspecto crítico, heredado de la antigua tópica, al sistema categorial. En ninguna otra obra se hace esto tan evidente como en la Critica a la Filosofia del Derecho, en la que la tarea crítico-refutativa avanza paso a paso, parágrafo por parágrafo, desnudando sus inconsistencias internas. Por supuesto, una tal crítica supone como punto de partida una actitud teórica diferente a la del objeto de la crítica. Lo que queremos aquí subrayar es que el punto de vista teórico del que parte la crítica es, considerado metódicamente, secundario con respecto al procedimiento mismo. Es un punto de vista a construir, justamente a través de la crítica misma y no como algo ya dado y fijo. 18

Las obras posteriores de Marx, desde La Sagrada Familia, pasando por La Ideologia Alemana hasta la Miseria de la Filosofia, pueden considerarse por una parte como la progresiva independización del método crítico, por otra, como su consecuente delimitación dentro del amplio campo antaño cubierto por la filosofía práctica al campo restringido de la historia social y de la economía politica.

3. Pero ¿qué forma específica de acuerdo con Marx adquiere la crítica desde el punto de vista metodológico en la discusión cientifica concreta? A fin de responder a esta pregunta daremos un ejemplo en el que Marx discute la teoría de A. Smith según la cual el trabajo es el precio real de las mercancias.

El texto de A. Smith es el siguiente:

Termini 'Analytik' und 'Dialektik'" Archiv für Begriffsgeschichte, VII (1962) pp. 120-139, esp. pp. 126 ss.

13 Cf. Tonelli, G. op. cit., pp. 129 ss.

14 Cf. W. Hennis, Politik und praktische Philosophie, Neuwied-Berlin: Luchterhand, 1963, pp. 35 ss y 89 ss.

15 Cf. sobre la relación de Hegel con la antigua dialéctica. H. G. Gadamer, "Hegel und die antike Dialektik", Hegelstudien, I (1961), pp. 173 ss. Véase también J. Habermas, Theorie und Praxis, Neuwied-Berlin: Luchterhand 1969, pp. 13-51, esp. 47 ss.

16 Cf. las afirmaciones de Marx sobre la critica en la Introducción a la Critica de la Filosofia del Derecho y en los Manuscritos económico-filosóficos, $M W$ I, pp. 488 ss; 637 ss. 
Cantidades iguales de trabajo puede decirse que tienen en todo tiempo y lugar el mismo valor para el trabajador. En su estado normal de salud, fuerza y actividad; en su grado ordinario de destreza y habilidad, él debe sacrificar siempre la misma porción de su ocio, de su libertad y felicidad. El precio que paga debe ser siempre el mismo, como quiera que sea la cantidad de bienes que recibe en cambio de él. En realidad, de éstos puede a veces adquirir una cantidad mayor, a veces una menor; pero es el valor de los bienes el que varía, no el del trabajo que los adquiere. (...) Sólo el trabajo no varía nunca en su propio valor y, por lo tanto, es exclusivamente el criterio último y real por el cual el valor de todas las mercancías puede ser estimado y comparado en todos los lugares y en todos los tiempos. Él es su precio real; el dinero es solamente su precio nominal. ${ }^{17}$

La discusión de este texto se encuentra en los Grundrisse y ocupa cuatro densas páginas de los mismos, razón por la cual daremos aquí sólo una pará. frasis de los principales argumentos:

El trabajo considerado simplemente como sacrificio y por ello como determinante del valor, como precio que se paga por las cosas y que les da por tanto un precio según la mayor o menor cantidad de trabajo que cuestan, es una determinación puramente negativa. Pero una determinación meramente negativa no crea nada. La negación del reposo como sacrificio ascético no crea de por sí nada. Un monje puede martirizarse todo el día y la cantidad de sacrificio que lleva a cabo no produce de por sí nada. El precio natural de las cosas no es, por lo tanto, el sacrificio que exigen. Sólo el trabajo produce; es, por lo tanto, la única substancia de los productos en tanto valores. La medida del trabajo, el tiempo del trabajo, supuesta una intensidad igual, es por tanto la medida de los valores. Dos cosas son conmensurables con la misma medida sólo si son de la misma naturaleza. Los productos pueden medirse con la medida del trabajo, el tiempo de trabajo, porque según su naturaleza son trabajo. Ellos son trabajo objetivado. La diferencia cualitativa de los trabajadores, esto es, lo expresado por la división del trabajo, por su diferenciación, es también un resultado histórico. La medida del trabajo, el tiempo, no depende de su productividad. Su medida no es más que una unidad, de la cual sus partes alicuotas expresan un determinado número. De ello no se sigue que el precio del trabajo sea constante, o solamente en la medida en que cantidades iguales de trabajo equivalen a unidades de medida iguales. El valor de los productos no es medido por la cantidad de trabajo aplicado en ellos, sino por la cantidad necesaria para su producción. Para el trabajador puede significar una hora de trabajo siempre el mismo sacrificio; el valor de las mercancias no depende en absoluto de sus sentimientos, como tampoco el valor de su

17 The Wealth of Nations, I ch. 5, ed. A. Skinner, Harmondsworth 1974, p. 136. 
hora de trabajo. Esto es concedido por A. Smith, quien señala que este sacrificio se puede comprar a veces más caro y a veces más barato. ${ }^{18}$

A fin de poder contraponer ambos argumentos, debemos reducirlos a un esquema común, que nos permita ver con claridad en qué reside el procedimiento de refutación. Hasta época muy reciente el olvido de la antigua tópica hacía imposible entender el funcionamiento interno de un argumento. Descartada, por inapropiada, la lógica formal, todo argumento material, esto es, no formal-deductivo, quedaba privado de todo sustento lógico. Con la aparición del libro de S. Toulmin, The Uses of Arguments ${ }^{19}$ se ha abierto la posibilidad de encarar esquemas argumentativos no formales, por un lado, y de volver a entender el mecanismo interno de la lógica de los tópicos, que permaneció vigente desde Aristóteles hasta el fin de la Edad Media, y su validez para la comprensión de la discusión científico-crítica, por el otro. EI esquema que vamos a desarrollar, si bien se basa en el propuesto por Toulmin, ${ }^{20}$ recoge la aplicación del mismo hecha por De Pater a la Tópica de Aristóteles. ${ }^{21}$

A. Smith:

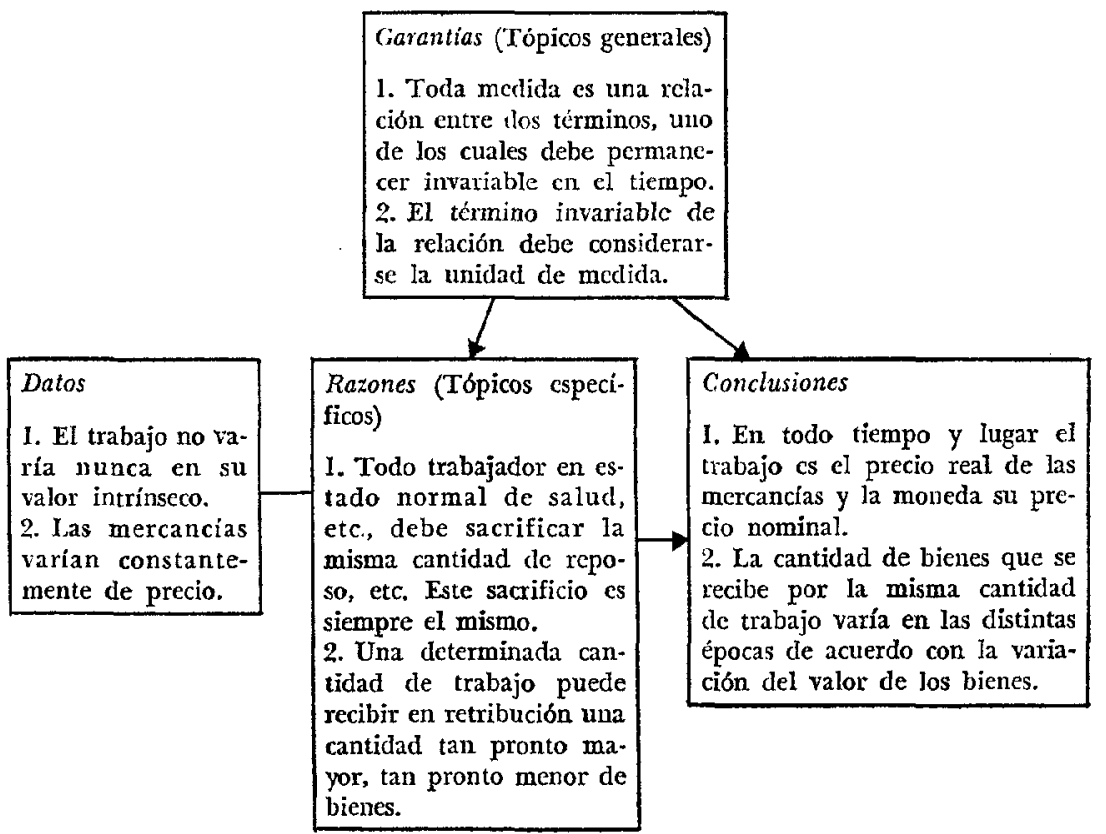

18 Grundrisse, pp. 504-508.

19 1a ed. Cambridge: U. P., 1958; $2^{\text {a }} 1964$.

20 Op. cit., c. III: "The Layout of Arguments", pp. 94 ss.

$21 \mathrm{~W}$. A. De Pater, Les Topiques d'Aristote et la dialectique platonicienne, Fribourg (Suisse) 1965, pp. 129 ss. 


\section{K. Marx:}

\begin{tabular}{|c|c|c|}
\hline & $\begin{array}{l}\text { Garantias (Tópícos generales) } \\
\text { 1. De una negación no se } \\
\text { sigue una afirmación (posi- } \\
\text { ción), a menos que sea una } \\
\text { negación de una negación. } \\
\text { 2. Si algo es un producto, } \\
\text { debe scr el producto de una } \\
\text { cierta actividad que lo pro-- } \\
\text { duce, de un cierto trabajo. } \\
\text { 3. Dos cosas son conmensu- } \\
\text { rables con la misma medida } \\
\text { sólo si son de la misma na- } \\
\text { turaleza. } \\
\text { 4. La productividad media } \\
\text { del trabajo depende del gra- } \\
\text { do de división y diferencia- } \\
\text { ción del trabajo histórica- } \\
\text { mente alcanzado. }\end{array}$ & $\begin{array}{l}\text { les) } \\
\text { se } \\
\text { osi- } \\
\text { na } \\
\text { cto, } \\
\text { ina } \\
\text { ro- } \\
\text { su: } \\
\text { ida } \\
\text { na- } \\
\text { dia } \\
\text { ca- } \\
\text { ca- } \\
\text { ca- }\end{array}$ \\
\hline $\begin{array}{l}\text { Datos } \\
\text { 1. Todo trabajador } \\
\text { en estado normal, } \\
\text { etc. debe sacrificar } \\
\text { la misma cantidad } \\
\text { de reposo, ctc. } \\
\text { 2. Una decermina- } \\
\text { da cantidad de tra- } \\
\text { bajo recibe una } \\
\text { cantidad mayor o } \\
\text { menor de bienes se. } \\
\text { gún las ćpocas. }\end{array}$ & $\begin{array}{l}\text { Razones (Tópicos especi- } \\
\text { ficos) } \\
\text { 1. El sacrificio de un } \\
\text { monje durante todo cl día } \\
\text { no produce ningún valor. } \\
\text { 2. Los bienes son produc- } \\
\text { to del trabajo, son ellos } \\
\text { mismos trabajo. } \\
\text { 3. La medida del trabajo } \\
\text { cs el tiempo. } \\
\text { 4. El grado de producti- } \\
\text { vilad de una misma can- } \\
\text { tidad de trabajo varia se- } \\
\text { gún el desariollo de los } \\
\text { instrumentos de trabajo } \\
\text { y de la división del tra- } \\
\text { bajo. }\end{array}$ & $\begin{array}{l}\text { Conclusiones } \\
\text { 1. El sacrificio no es la medida } \\
\text { del valor de las mercancias. } \\
\text { 2. El tiempo de trabajo o can- } \\
\text { tidad de trabajo es la medida } \\
\text { del valor. } \\
\text { 3. El valor del trabajo no es } \\
\text { constante (a menos que se quie- } \\
\text { ra expresar una tautología igua- } \\
\text { les cantidades de trabajo son } \\
\text { cantidades iguales de la umidad } \\
\text { de medida por la que se mide } \\
\text { el trabajo). } \\
\text { 4. Los valores de los productos } \\
\text { son medidos no por el trabajo } \\
\text { aplicado a ellos, sino por el tra- } \\
\text { bajo socialmente necesario para } \\
\text { la producción de los mismos. } \\
\text { 4.1. Aunque la cantidad de tra- } \\
\text { bajo sea la misma, puéde variar } \\
\text { su productividad y por tanto su } \\
\text { valor. }\end{array}$ \\
\hline
\end{tabular}

El funcionamiento del esquema, tal como lo presentamos, es el siguiente:

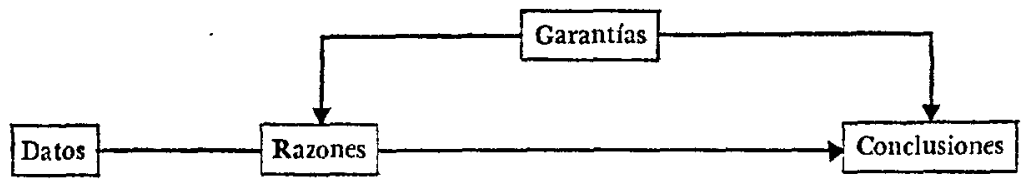


Los datos (D) son los datos indispensables en los que se plantea el problema. Las razones ( $R$ ) equivalen a los datos que, en el esquema de Toulmin, respaldan las garantías (G). Aquí se las ha interpretado de un modo un tanto diferente. Se trata de razones de tipo específico, más generales que los datos, pero ligadas a éstos, de modo que, unidas a los datos mismos, permiten extraer la conclusión correspondiente. Ahora bien: la conclusión sólo puede extraerse mediante una regla de inferencia que permita el paso de $D$ y $R$ a $C$. Esta regla de inferencia es, creemos, de carácter más general que la señalada por Toulmin y corresponde más bien al tópos koinós de Aristóteles, esto es, a la formulación más general posible unida aún al significado material de los términos. A modo de ejemplo tomemos la G 2 del esquema de Marx. Se trata, en última instancia, de una forma particular del tópos o lugar general de los contrarios, en este caso de los contrarios relativos, que afirma que la posición de un relativo implica la posición de su correlato: la posición de amo implica la posición de esclavo. Se puede pensar que, como señala Bird, la $G$ asi tomada se aproxima hasta confundirse con un teorema de lógica formal, en este caso de la lógica de relaciones. ${ }^{22}$ El punto que nos interesa señalar es que ningún teorema formal cumple con la función específica de la $G$, esto es: asegurar que la conclusión pasible de ser extraída de esos datos y de esas razones es la efectivamente extraida y a la inversa, que para extraer tal conclusión se requerían precisamente esos $D$ y esas $R$, y no otros. Es éste el carácter práctico de la $G$, señalado por Toulmin, que rescata un viejo postulado de la Identitätsphilosophie del idealismo alemán, heredado a su vez de Kant, y que podemos resumir así: en toda conexión entre dos términos empíricos se debe suponer la existencia de un acto previo de la razón, que establece, por una parte, la conexión, la sintesis de los datos y la conclusión, y que garantiza, por otra, que tal conexión es admisible. ${ }^{23}$

El razonamiento de Marx es extremadamente complejo; el esquema propuesto por nosotros ayuda a entender el porqué de esa complejidad. Marx toma como datos (D) las razones (R) expuestas por A. Smith, las cuales pro cederá a refutar o a interpretar de otra manera. Dado el esquema argumentativo, refutadas, en efecto, las razones sobre las que se apoyan específicamente los datos y que conducen a la conclusión, el argumento se derrumba. Dicho de otra manera, las razones y las garantías son el punto de soporte de los argumentos; si se demuestra su error, el argumento está irremediablemente perdido. Pero al par que refuta la $\mathrm{R} 1$ del argumento de Smith, Marx introduce razones propias para resolver el mismo problema que Smith no pudo resolver. Marx explicita dos tópicos generales, G 1 y 3, que han sido violados o no tenidos en cuenta en el argumento de A. Smith. En efecto, la R 1 de Smith supone una $G$, no explicitada, de carácter general contraria a la $G 1$ asentada por Marx. Esta se apoya y apoya a su vez la $R \mathbf{l}$, que es la afirmación perentoriamente refutativa: el sacrificio de un monje no por ser sacrificio produce algún valor. Algo un tanto distinto ocurre con la G 3 de Marx.

22 Cf. O. Bird, "The Re-discovery of the "Topics'", Proc. of the Amer. Cathol. Phil. Asoc. 34 (1960) pp. 200-205.

23 Kant, K.r.V. B 129 ss. 
En realidad viene a ser una condición general de carácter ontológico que limita la vigencia de G 1 y 2 de A. Smith: admitidos los tópicos generales tácitamente supuestos de A. Smith respecto de la medida, para que tengan validez, los términos de la relación deben ser de la misma naturaleza. Distinto es el argumento iniciado con el D 2 de Marx. Lo que A. Smith da como $R$, es en realidad un problema, no una $R$ que permita una inferencia. El carácter de la argumentación va a variar sensiblemente en este argumento que corre paralelo al primero. Si comparamos el D 2 de Marx con las conclusiones 2 a 4, veremos que el contenido de las últimas es esencialmente el mismo que el del dato. El fin de la argumentación no reside, en efecto, en establecer una nueva conclusión, sino en descubrir las $R$ y $G$ que permiten comprender el problema (D) en términos más amplios (C). Se trata de una forma especial de argumentos, warrant-establishing en la terminologia de Toulmin, ${ }^{24}$ es decir, que establecen $G$. Es ésta una forma típicamente marxiana de exploración, que consiste en partir de un dato, ponerlo en suspenso como dato a fin de buscar la razón general que permita afirmarlo en la conclusión nuevamente como dato, pero ahora en una forma general y comprensible. No nos vamos aquí a extender sobre este punto, que forma parte de lo que se ha llamado la "Iógica de la esencia" en Marx.. ${ }^{25}$ Señalemos únicamente que lo importante de este carácter del pensamiento de Marx al volcarse en el molde de la discusión cientifica, es su capacidad de desnudar $R$ y $G$ supuestas allí donde otros no ven sino datos. Demás está decir, por último, que lo decisivo en esta argumentación de Marx es el descubrimiento de la $\mathrm{R} 2$ (los bienes son trabajo) y de la G 2 (el producto es una objetivación de la actividad que lo produce), que permite obtener la $\mathrm{C} 2$, es decir, la teoria marxiana del valor.

No estaría completo el cuadro de la lógica investigativa de Marx si no quedara en claro qué queda fuera de ella, esto es, el conjunto de convicciones que acompañan y fijan el rumbo de la discusión. Marx inicia la discusión por ellas, señalando su carácter filosófico y no económico. Se trata del concepto negativo del trabajo en Smith, de su carácter de sacrificio y dolor. Para Marx, en cambio, este carácter fáctico, histórico, del trabajo, que proviene de su condición de enajenado, oculta su aspecto positivo, de autoafírmación del hombre, aspecto que alguna vez se abrirá paso hacia la luz. Rozamos con ello el problema de la ideología y de la crítica de la ideología, que aquí apenas podemos esbozar. Así como los datos, razones, garantías y conclusiones torman un sistema cerrado que se apoya mutuamente, lo cual, a su vez, tiende a individualizar un argumento y darle un carácter propio, de la misma manera la elección de determinadas garantías dentro de las infinitas posibles está en estrecha relación con la orientación total del pensamiento, con su intencionalidad. Como se ha señalado más arriba, las reglas de inferencia que constituyen las garantías sólo pueden cumplir con esa función en virtud de su carácter práctico, o dicho de otra manera, en virtud de que en ellas se afirma y manifiesta la naturaleza práctica de la razón. Admitir la naturaleza prác-

24 Op. cit., p. 120.

25 Cf. la excelente exposición de J. Zeleny, Die Wissenschaftslogik und "Das Kapital", Frankfurt-Wien: Europa, 1969, esp. pp. 35 ss. 
tica de la razón, al menos en el ámbito de las ciencias sociales, significa admitir al mismo tiempo la vigencia de fines hacia los cuales la razón está orientada y para cuyo cumplimiento recurre a mediaciones, esto es, a juicios e inferencias como las estudiadas. Dado que éste es el carácter constitutivo de la razón en el ámbito práctico, la objetividad no consiste en negarlo, sino en explorar abiertamente los límites dentro de los cuales una discusión científica puede tener aún lugar y las posibilidades de extender más allá de este límite el consenso mediante comprensión y persuasión. También en este aspecto la discusión de Marx con A. Smith es ejemplar.

Centro de Investigaciones Filosóficas

Osvaldo N. Guariglia Buenos Atres, Argentina

\section{REFERENCIAS}

MEW K. Marx-F. Engels, Werke, ed. por el Institut f. Marx. del SED, Berlin: Dietz, 1956 ss.

MW K. Marx, Werke, ed. por H. J. Lieber y colab., Darmstadt: Wissensch. Buchgesell., 1962 ss. 